\title{
Dynamic Glyphs: Appropriating Causality Perception in Multivariate Visual Analysis
}

\author{
Khairi Reda* $\quad$ Caleb Potts $\quad$ Taylor Childers
}

Indiana University-Purdue University Indianapolis
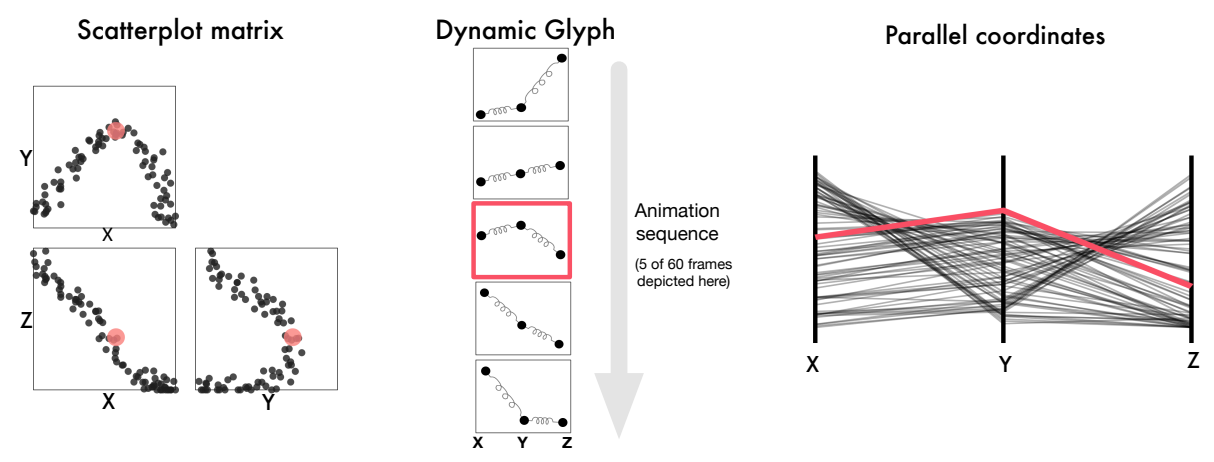

Figure 1: A 3-variate dataset shown using a scatterplot matrix, parallel coordinates plot, and our proposed Dynamic Glyphs approach (center). We visualize multivariate datasets using flipbook-style animation, where each frame represents one data point. Glyph features depict attribute values (here encoded in the height of 'beads'). Enactment of the animation produces motion in glyph parts, creating perceptual impressions of causality. These causal percepts aids the observer in seeing the underlying multivariate interactions, more so than with static alternatives.

\begin{abstract}
We investigate how to co-opt the perception of causality to aid the analysis of multivariate data. We propose Dynamic Glyphs (DyGs), an animated extension to traditional glyphs. DyGs encode data relations through seemingly physical interactions between glyph parts. We hypothesize that this representation gives rise to impressions of causality, enabling observers to reason intuitively about complex, multivariate dynamics. In a crowdsourced experiment, participants' accuracy with DyGs exceeded or was comparable to non-animated alternatives. Moreover, participants showed a propensity to infer higher-dimensional relations with DyGs. Our findings suggest that visual causality can be an effective 'channel' for communicating complex data relations that are otherwise difficult to think about. We discuss the implications and highlight future research opportunities.
\end{abstract}

\section{INTRODUCTION}

Visualization maps data attributes to perceptible features that can be processed by our visual system. Designers select from a variety of properties, such as the position of points, the length of bars, or the motion speed of objects. These (and other) elementary marks and channels are often considered the foundational building blocks for visualization $[25,41]$. Our vision, however, is not limited to analyzing basic geometric features or their surface properties. In fact, our visual system also works to subconsciously infer higher-level relationships, which are rarely leveraged in visualization design.

One relationship that captures our attention is causality. In its simplest form, visual causality presents as two objects interacting physically, with one object affecting a change in the other. For example, a rolling billiard ball accelerates towards a second, initially stationary ball. Upon collision, the moving balls imparts its

*e-mail: redak@iu.edu kinetic energy onto the second, causing it to move. This sort of visual sequence, known as a "launching event", has been studied extensively by psychologists [23]. Unsurprisingly, observers of launching events can quickly recognize the underlying causal relationship (i.e., the still ball had moved in reaction to the moving ball). However, what makes this kind of visual phenomenon interesting for visualization: the fact that the impression of causality results from perceptual, unconscious processes [36,37]. Put simply, our visual system appears to combine the various spatio-temporal features that comprise a launching (or a similar) event. It then makes the underlying causality available as a unit perceptual feature.

Causality perception is thought to originate in obligatory processes that are independent from knowledge or experience [8,12,34]. Remarkably, infants as young as 7 months old [20,21,26] and Chimpanzees $[22,28]$ seem predisposed to recognizing and reacting to visual causalities. Causal events thus represent a class of elementary visual features that our brains have evolved to process preattentively-perhaps in ways that are not unlike conventional channels such as color, size, and shape [24]. In short, perceptual theory suggests that causality can be a modular and intuitive cue [37], but this 'channel' remains largely untapped in data visualization.

In this paper, we ask the question: can we appropriate people's perception of causality to highlight relations in data that might otherwise be difficult to comprehend? In particular, we consider the analysis of multivariate data (i.e., datasets that involve relationships between three or more variables), which are notoriously difficult to analyze with existing techniques [45]. Visualizing such relations as physical causalities could help make them more intuitive. The challenge, however, is to come up with an evocative design that adheres to the 'laws' of causality perception while being malleable to representing arbitrary datasets.

We propose Dynamic Glyphs (DyGs), an animated extension to traditional multivariate glyphs that is loosely modeled after parallel coordinates (see Fig. 1-center). This extension allows the glyph to morph as it displays different data points in a flipbook-style animation. We propose a series of optimizations to maximizing the glyph's

This is the author's manuscript of the article published in final edited form as:

Reda, K., Potts, C., \& Childers, T. (2019). Dynamic Glyphs: Appropriating Causality Perception in Multivariate Visual Analysis. https://doi.org/10.31219/osf.io/s5q9n 
spatio-temporal contiguity. We hypothesize that these optimizations, together with other visual cues, give rise to a perceptual experience of causality. This in turn should make it easier for observers to see and describe complex data relationships in terms of physical interactions between glyph parts. In a crowdsourced study, we compare DyGs against two non-animated (but interactive) visualizations: scatterplot matrices (SPLOM) and parallel coordinates plots (PCP) We find that participants' accuracy with DyGs exceeds or is comparable to non-animated alternatives. Moreover, participants showed a propensity to infer higher-dimensional data relations with DyGs, as compared with SPLOM and PCP. Our findings suggest a new avenue for incorporating causally cued motion into data visualizations, so as to enhance the saliency and communicability of complex patterns.

\section{BACKgROUND \& RELATED WORK}

Multivariate datasets routinely arise in a range of domains, from business and commerce to science and engineering. The chief challenge in these datasets is to discover how the attributes interact and affect each other [45]. Such analysis can be challenging; the analyst need not only look for bivariate trends, but also has to consider the potential for interactions between three or more factors.

Arguably, the most familiar way to visualize multivariate data is to use scatterplots [30]. Scatterplots can be highly effective, especially if the intent is to look for bivariate correlations [13,32]. However, they are practically limited to displaying 2-3 variables at a time. To cover all data dimensions, one would need to look at a scatterplot matrix (SPLOM) [5]. SPLOMs allow one to easily see all possible bivariate relationships. However, in doing so, they relatively impede the perception of larger relations spanning multiple variables. As an example, consider the SPLOM depicted in Fig. 1-left. The observer here can easily view the quadratic relationships in the $X, Y$ and $Y, Z$ plots. He/she should also have no problem observing the inverse correlation between $X$ and $Z$. However, the observer might miss a less salient but important feature here: that the infliction points in the two quadratic relations coincide with $Z$ reaching its mid-level height (highlighted in red). Put differently, it is as if $X$ and $Y$ correlate positively as long as $Z$ is above a certain value. Falling beyond this threshold, $Z$ is associated with a decoupling of the linear relationship between $X$ and $Y$, with the latter then becoming more correlated with $Z$.

The above trivariate interpretation might be interesting for the analyst to ponder. Such relation can only be understood in its entirety; perceiving the three scatterplots individually is not enough for one to realize how the variables interact in unison. We argue that this a problem for existing visualizations, including SPLOMs, parallel coordinates [16], and traditional glyphs [4]. Even with brushingand-linking [3], it can be challenging for analysts to observe and think about multivariate trends in disjoint views. Consider, instead, the visualization at the center of Fig. 1, which represents the same data but employs a flipbook-style animation (only 5 of the 60 frames shown in the figure). Here, $X, Y, Z$ are encoded in the vertical positions of three 'beads'. The progression of the animation illustrates how $X$ and $Y$ rise together, all the while $Z$ is falling (top 3 frames). The animation then shows how the positive correlation between $X$ and $Y$ begins to break down when $Z$ reaches a threshold (bottom 2 frames). At that point, $Y$ stops rising and begins to fall instead, as if it is being dragged down by $Z$.

\subsection{Animation in Data Visualization}

Motion has been used sparingly in multivariate visual analysis. Its use has been primarily restricted to animated transitions [14]. For instance, ScatterDice allows smooth transition between projections by interpolating on one axis at time [10]. Yi et al. describe a 'dust \& magnets' metaphor, which allows an analyst to construct custom $2 \mathrm{D}$ projections by placing magnets (corresponding to attributes) and observing how the point cloud reacts [46]. However, the use of

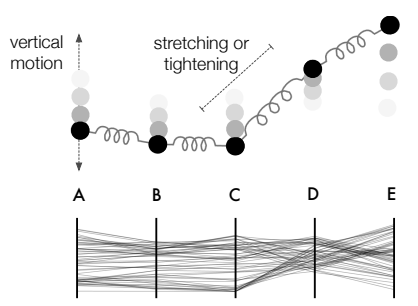

Figure 2: A composite of a Dynamic Glyph animation (top) and its equivalent parallel coordinates plot. The glyph shows a multivariate point using one bead for each attribute. Beads move vertically to indicate higher or lower attribute values. Springs connect the beads and react to their motion by stretching and tightening

motion in InfoVis has come with some controversy. Robertson et al. tested animated scatterplots (in the style of GapMinder [35]) but found them less accurate than static plots and small-multiples [33]. Similarly, Tversky et al. suggest that any benefits of animation can be realized by supplanting dynamic graphics with a series of static views [40]. On the other hand, recent work have demonstrated effective uses for animation. For instance, animated graphs of dynamic networks tested more accurate than small-multiples [1]. Ondov et al. also found animation to be more effective for outlier detection [29]. Animation can induce a frequency-based framing of uncertainty [15], which seems advantageous over static representations [18].

Researchers have used motion to highlight causal relationships [2, $11,17,42]$. A key assumption in these works is that causalities are known a priori, with visualizations intended to present a summary thereof. Our approach is to let the viewer perceptually deduce visual causality from a 'playback' of data frames, without necessarily implying causality in the epistemological sense [31]. Our technique is therefore broadly applicable to multivariate data, and not restricted to communicating known causalities - a much narrower context.

\section{Dynamic Glyphs}

We aim to design animated devices that can leverage people's innate causality perception. We call these representations Dynamic Glyphs (DyGs), as they are inspired by the classical glyph notion. A glyph consists of several marks each mapped to a separate data attribute. The visual properties of the marks (e.g., the position of circles or the length of bars) are determined by data values. Howver, unlike traditional glyph designs where different data points are encoded with separate visual objects, DyGs employ a single glyph that is animated over a sequence of frames. Each frame depicts a single multidimensional data point. Collectively, the animation depicts an entire dataset, illustrating interactions between attributes via the motion of glyph parts. For this animation to be effective, however, it must be optimized.

\subsection{Optimizing for Causality Perception}

The first challenge is to design a glyph that, when animated, can potentially evoke a sense of physical interactions between its constituent parts. That is, the marks should be seen as exerting forces on each other. We adopt the glyph in Fig. 2 as an initial prototype. The glyph is composed of a series circular beads, one for every data attribute to be encoded. The value for an attribute is encoded in the vertical position of the bead. Beads move along parallel vertical axes as the glyph transitions between different data points. A correlation between attributes thus can be seen as beads moving in unison. To convey a potential for physical interactions, adjacent beads are connected with coil springs. The springs do not exert influence on the position of the beads, as the latter is driven entirely by the data. Rather, springs follow the movement of beads, and stretch and tighten in reaction. While completely reactionary, the 


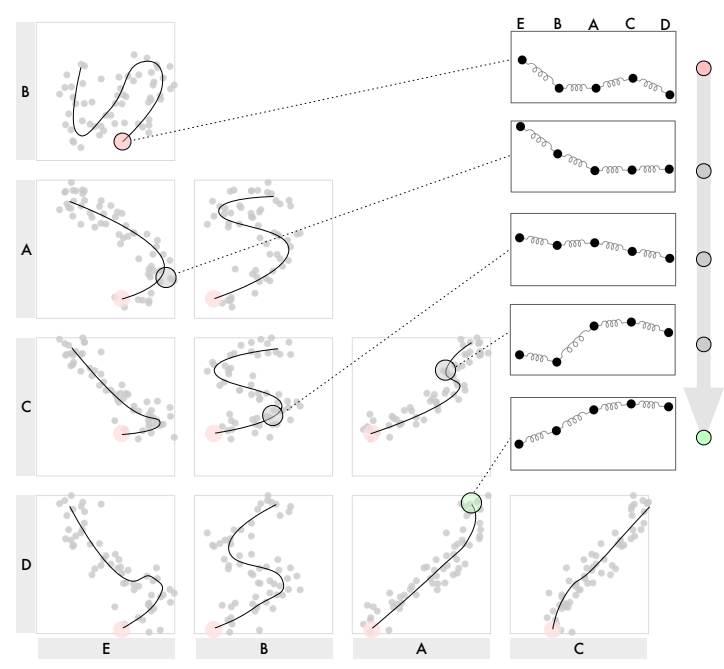

Figure 3: Optimization of Dynamic Glyphs animation. We determine a frame sequence (illustrated with curves in the bivariate projections) by minimizing Euclidean distance between consecutive frames. Correlating attributes (e.g., A, C, D) are also mapped to adjacent beads.

springs serve to communicate a notion of implied forces between the attributes. One caveat with this design is that the nature of forces implied (i.e., pulling via spring tension) differ from the kind of collision forces seen in causality studies $[23,36]$. However, evidence suggests that causal percepts can also be triggered when the motion suggests pushing and pulling [44], even with no direct contact [43].

The second challenge is to make the animation seem physically plausible. This is essential, as causal perception requires spatiotemporal contiguity in the motion [6,23]. We address this by first optimizing the order of attributes within the glyph to maximize bivariate correlation between neighboring beads. That is, highly correlated attributes are mapped to adjacent beads whereas those showing no association are placed further apart. This strategy should increase spatial contiguity. Temporal contiguity, on the other hand, can be optimized by 'playing' similar frames consecutively (recall that each frame in the animation corresponds to one data point). We thus compute a sequence that minimizes the intra-frame Euclidean distance, and where every data point appears exactly once. Fig. 3 illustrates the intuition behind this optimization.

These two optimizations should not impact data semantics, as the order of columns or rows within a multivariate dataset do not carry any meaning. To further increase motion coherence, we apply a moving average so that the shape of the glyph at a particular frame is dependent to some degree on its three prior and subsequent frames.

\section{EXPERIMENT}

We conducted a crowdsourced experiment to test whether DyGs can help people find and report multivariate relations. We also compare DyGs against two traditional alternatives: scatterplot matrices (SPLOM) and parallel coordinates (PCP). The experiment utilized the following protocol: participants were shown a stimulus multivariate dataset using one of the three visualizations. For each stimulus, we asked the participant to describe apparent data relations in natural language [27]. This open-ended response is meant to capture participants' spontaneous interpretations of data with minimal bias.

\subsection{Stimuli}

We opted for synthetic stimuli in order to generate datasets with known ground truths. This was done by first sketching bivariate trends in $2 \mathrm{D}$ scatterplots and randomly adding points around the sketch. The sketched relations could then be generalized to three or more dimensions by drawing in multiple plots corresponding to different attribute pairs. This process enabled us to embed meaningful ground-truths in the stimulus set, while balancing three factors:

- Dimensionality: we generated stimuli with 3, 4, or 5 variables.

- Degrees of Freedom (DoF): we sought to vary the degree to which the dimensions correlate in the stimuli. We quantified the DoF by running a PCA analysis on the stimuli. Larger DoF reflects more variance among the dimensions, and hence higher data complexity.

- Data features: We sought to vary the features within the stimuli by embedding linear correlations, polynomial relations, clusters within bivariate projections, and random bivariate distributions.

We generated 12 datasets as stimuli for the experiment. Fig. 3 shows one of the 5-dimensional stimuli tested. Each dataset consisted of 60 multidimensional points. Attribute values were normalized unitless real numbers. We refrained from giving the attributes semantically resonant names to avoid biasing participants [9]. Instead, attributes were labeled with single letters (A through E) so that participants can refer to them unambiguously. Consult the supplemental materials for details ${ }^{1}$.

\subsection{Visualizations}

Participants saw one of three visualizations (Fig. 1):

- Dynamic Glyphs: The glyph transitioned between data points in a loopy animation. In keeping with the illustrative nature of DyGs, no axes, quantities, or units were shown - only relative motion of the beads and springs can be inferred (Fig. 2). The duration of the animation was 1700 milliseconds (yielding 34 data frames per second). The interface included a pause/replay button and an interactive progress slider, allowing participants to jump frames.

- SPLOM: The display showed the lower half of the scatterplot matrix. Participant could also brush data points in one scatterplot and see them highlighted in other plots.

- PCP: Parallel coordinates can be seen as orthogonal to DyGs; attribute values are encoded with vertical positions on parallel axes. However, PCP avoids animation by depicting all data points at once via semi-translucent, brushable polylines.

\subsection{Participants \& Procedures}

We recruited 123 participants from Amazon Mechanical Turk (42 females, 80 males, 1 unspecified) with a mean age of 33.74 (SD=11.32 years). Participants were randomly assigned to one of the three visualization types, with 41 subjects in each condition. All participants viewed the same 12 synthetic datasets ( $\$ 4.1)$. Stimuli were ordered based on the dimensionality of the dataset (low to high). After a brief tutorial, participants viewed the stimulus datasets one at a time. For each dataset, participants were asked to respond to the following prompt (inspired by [36]): Your task is to describe what is happening in these charts and what the attributes are doing. We are interested in your intuitive impressions. Do you have an impression that some attributes are somehow interacting with other attributes? Please refer to attributes specifically by their symbol. Participants typed their open-ended response in a text box. Subjects were compensated with a $\$ 2$ payment upon completing the study.

\subsection{Analysis and Coding}

We segmented participants' responses [39] to delineate observations relating to distinct data features. Each segment was then tagged with one or more codes characterizing the data feature implied (see the supplemental materials for examples). We extracted two quantitative metrics from each segment. First, the accuracy of implied data features was determined by comparing responses against the ground truth. Accordingly, segments were graded as 1 (correct) or 0 . Segments that could be interpreted as partially correct, or those that needed a more nuanced description (e.g., when characterizing

${ }^{1}$ The supplemental materials are available at: https://osf.io/qv6d2/ 

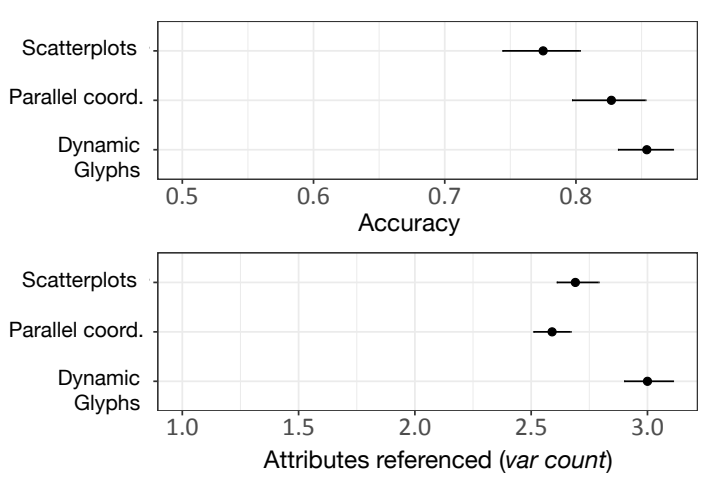

Figure 4: Average accuracy (top chart) and number of uniquely referenced attributes (bottom) in participants' responses $( \pm 95 \% \mathrm{Cls})$.

a nonlinear relationship as a simple correlation) were graded as $\frac{1}{2}$. Segments that could not be graded due to lack of specificity were excluded from the analysis. We extracted a second metric that reflected the number of unique attributes referenced in each segment (hereafter var count). This number captures the complexity of the inferred patterns and the degree to which they involve multiple variates. For instance, in the segment " $\mathrm{A}$ and $\mathrm{C}$ go down and then increase as B increases", the rater counted 3 unique attributes.

Two raters redundantly coded 137 responses (9.5\% of total). Interrater agreement was computed using Fisher's Intraclass Correlation Coefficient (ICC) [19]. The observed ICC was 0.711 and 0.82 for accuracy and var count, respectively, indicating good agreement [7].

\subsection{Accuracy}

Fig. 4-top illustrates the mean response accuracy. Results did not meet the normality assumption, so we employ a non-parametric Kruskal-Wallis test. The test indicates a significant main effect of visualization type $\left(\chi^{2}(2)=19.596, p<0.001\right)$. A Mann-Whitney U test with Bonferroni correction shows significant difference between DyGs and SPLOM $(p<0.001)$, and between SPLOM and PCP $(p<0.001)$. No difference was found between DyGs and PCP. The results suggest a comparable accuracy for Dynamic Glyphs $(85.4 \%)$ and parallel coordinates $(82.7 \%)$, both of which seemed more accurate than scatterplots $(77.5 \%)$.

\subsection{Response Complexity}

Fig. 4-bottom illustrates the average number of attributes referenced by participants in their responses. A Kruskal-Wallis test reveals a significant main effect of visualization type on the number of attributes referenced $\left(\chi^{2}(2)=43.332, p<0.001\right)$. Post-hoc MannWhitney tests show significant difference between DyGs and PCP $(p<0.001)$, and between DyGs and SPLOM $(p<0.001)$. Dynamic Glyphs led to more complex multivariate inferences (involving 3 attributes on average) compared to parallel coordinates (2.58) and scatterplots (2.69), with the latter two being comparable.

\section{Discussion}

Results show Dynamic Glyphs (DyGs) to be more accurate than scatterplot matrices (SPLOM). This seems surprising at first, given that scatterplots are known to be effective at showing bivariate trends $[13,32]$. However, unlike individual scatterplots, a SPLOM can be challenging to interpret, especially when looking for relationships between multiple variable. For example, generalizing bivariate trends to three (or more) dimensions requires careful inspection of several plots while brushing-and-linking. These steps can be cognitively demanding and may be beyond the skills of the typical analyst. By comparison, DyGs illustrate relations as visually causal interactions. This representation is arguably more intuitive, and may activate innate perceptual and cognitive mechanisms. The lower SPLOM accuracy may also be due to participants' occasional sloppiness when interpreting scatterplots. We frequently saw responses that characterize an inverse correlation (e.g., between $X$ and $Z$ in Fig. 1) as "two [variables] decreasing"—an obviously incorrect inference. These participants appear to confuse scatterplots with line charts.

The experiment shows that PCP and DyGs have comparable accuracy. This may be explainable considering the similarity between the two visualizations; both DyGs and PCP encode values as vertical positions on parallel axes. Overall, it seems Dynamic Glyphs can be at least as accurate as traditional multivariate visualizations.

The results, however, show a larger effect on the complexity of inferences. Participants seemed more likely to report higherdimensional patterns with DyGs, as measured by the number of attributes. This could be due to DyGs activating causal perception processes, thereby making these patterns more salient. One particular advantage of causal impressions over other channels is that they are intuitively 'transitive'. To illustrate this, consider a DyG that is composed of three connected beads: A, B, and C. If one observes A to impart its 'energy' onto $\mathrm{B}$, with $\mathrm{B}$ moving in reaction and in turn affecting movement in $\mathrm{C}$, then it can be concluded-perhaps at a perceptual level - that the triplet are related. It is relatively easy with DyGs to see when multiple attributes 'transmit' energy over a chain, which may in turn help in recognizing higher-order patterns. By contrast, the bivariate inferences people tend to draw from SPLOM and PCP constitute isolated chunks, requiring significant mental and interaction effort (e.g., brushing) to integrate.

Another advantage for DyGs is that they afford narrative-centric accounts of multivariate data. For instance, earlier in the paper we characterized relations in Fig. 1 using a short narrative: $X$ and $Y$ rising together, followed by a shift in the relationship when $Z$ reaches a threshold. Likewise, we noticed that many participants had responded with similar accounts. Descriptions of DyGs often contained story elements, with subjects characterizing attributes as actors with agency. However minimal, such narratives could make it easier for people to reason and talk about complex data dynamics.

Although we see qualitative evidence that DyGs promote causal impressions (as intended with the design), this study alone cannot attribute the effects observed to causality perception per se [36]. It may be that the physical metaphor in DyGs, or the ability to frame multivariate relations in terms of simple narratives, were the primary drivers behind participants' improved comprehension. Future studies could attempt to tease out the individual or compound effects of these factors. It is also important to note that priming people to think causally may not always be desirable, despite the potential affordances this sort of reasoning brings. In particular, there is a risk of analysts confusing simple correlation with causality after viewing DyGs. Such risk could be alleviated by reminding analysts that causality - in the knowledge-based sense — should not immediately ascertained, despite what the metaphor might imply. Lastly, as with other dynamic representations, DyGs could cause people to miss or neglect certain relations due to inattentional blindness [38], a possibility that should be studied.

\section{CONCLUSION}

Impressions of causality are products of early vision processes. We sought to leverage these mechanisms in multivariate data analysis. To that end, we designed a new kind of animated representation called Dynamic Glyphs (DyGs), and found them to be as accurate as traditional visualizations. Furthermore, untrained observers seemed more likely to report complex, higher-dimensional relationships with DyGs than with other techniques. Our findings suggest that visual causality may be an effective 'channel' in multivariate visualization.

\section{ACKNOWLEDGEMENTS}

This work was supported in part by NSF award 1755611. 


\section{REFERENCES}

[1] D. Archambault, H. Purchase, and B. Pinaud. Animation, small multiples, and the effect of mental map preservation in dynamic graphs. IEEE Transactions on Visualization and Computer Graphics, 17(4):539-552, 2011.

[2] L. Bartram and M. Yao. Animating causal overlays. In Computer Graphics Forum, vol. 27, pp. 751-758. Wiley Online Library, 2008.

[3] R. A. Becker and W. S. Cleveland. Brushing scatterplots. Technometrics, 29(2):127-142, 1987.

[4] R. Borgo, J. Kehrer, D. H. Chung, E. Maguire, R. S. Laramee, H. Hauser, M. Ward, and M. Chen. Glyph-based visualization: Foundations, design guidelines, techniques and applications. In Eurographics (STARs), pp. 39-63, 2013.

[5] D. B. Carr, R. J. Littlefield, W. Nicholson, and J. Littlefield. Scatterplot matrix techniques for large n. Journal of the American Statistical Association, 82(398):424-436, 1987.

[6] H. Choi and B. J. Scholl. Measuring causal perception: Connections to representational momentum? Acta Psychologica, 123(1-2):91-111, 2006

[7] D. V. Cicchetti. Guidelines, criteria, and rules of thumb for evaluating normed and standardized assessment instruments in psychology. Psychological assessment, 6(4):284, 1994.

[8] J. De Freitas and G. A. Alvarez. Your visual system provides all the information you need to make moral judgments about generic visual events. Cognition, 178:133-146, 2018.

[9] E. Dimara, A. Bezerianos, and P. Dragicevic. Narratives in crowdsourced evaluation of visualizations: A double-edged sword? In Proceedings of the 2017 CHI Conference on Human Factors in Computing Systems, pp. 5475-5484. ACM, 2017.

[10] N. Elmqvist, P. Dragicevic, and J.-D. Fekete. Rolling the dice: Multidimensional visual exploration using scatterplot matrix navigation. IEEE Transactions on Visualization and Computer Graphics, 14(6):1539_ 1148, 2008.

[11] N. Elmqvist and P. Tsigas. Animated visualization of causal relations through growing 2d geometry. Information Visualization, 3(3):154172, 2004.

[12] J. A. Fugelsang, M. E. Roser, P. M. Corballis, M. S. Gazzaniga, and K. N. Dunbar. Brain mechanisms underlying perceptual causality. Cognitive brain research, 24(1):41-47, 2005.

[13] L. Harrison, F. Yang, S. Franconeri, and R. Chang. Ranking visualizations of correlation using weber's law. IEEE Trans. Vis. Comput. Graph., 20(12):1943-1952, 2014.

[14] J. Heer and G. Robertson. Animated transitions in statistical data graphics. IEEE Transactions on Visualization and Computer Graphics, 13(6):1240-1247, 2007.

[15] J. Hullman, P. Resnick, and E. Adar. Hypothetical outcome plots outperform error bars and violin plots for inferences about reliability of variable ordering. PloS one, 10(11):e0142444, 2015.

[16] A. Inselberg. The plane with parallel coordinates. The visual computer, 1(2):69-91, 1985.

[17] N. Kadaba, P. Irani, and J. Leboe. Visualizing causal semantics using animations. IEEE Transactions on Visualization and Computer Graphics, 13(6):1254-1261, 2007

[18] A. Kale, F. Nguyen, M. Kay, and J. Hullman. Hypothetical outcome plots help untrained observers judge trends in ambiguous data. IEEE Transactions on Visualization and Computer Graphics, 25(1):892-902, 2019.

[19] T. K. Koo and M. Y. Li. A guideline of selecting and reporting intraclass correlation coefficients for reliability research. Journal of chiropractic medicine, 15(2):155-163, 2016.

[20] A. M. Leslie. The perception of causality in infants. Perception, 11(2):173-186, 1982.

[21] A. M. Leslie and S. Keeble. Do six-month-old infants perceive causality? Cognition, 25(3):265-288, 1987.
[22] T. Matsuno and M. Tomonaga. Causal capture effects in chimpanzees (pan troglodytes). Cognition, 158:153-164, 2017.

[23] A. Michotte. The perception of causality (English translation). Basic Books, 1963.

[24] P. Moors, J. Wagemans, and L. de Wit. Causal events enter awareness faster than non-causal events. PeerJ, 5:e2932, 2017.

[25] T. Munzner. Visualization Analysis and Design. AK Peters Visualization Series. CRC Press, 2015.

[26] G. E. Newman, H. Choi, K. Wynn, and B. J. Scholl. The origins of causal perception: Evidence from postdictive processing in infancy. Cognitive psychology, 57(3):262-291, 2008.

[27] C. North. Toward measuring visualization insight. IEEE computer graphics and applications, 26(3):6-9, 2006.

[28] S. O'Connell and R. Dunbar. The perception of causality in chimpanzees (pan spp.). Animal Cognition, 8(1):60-66, 2005.

[29] B. Ondov, N. Jardine, N. Elmqvist, and S. Franconeri. Face to face: Evaluating visual comparison. IEEE Transactions on Visualization and Computer Graphics, 25(1):861-871, 2019.

[30] M. J. Pastizzo, R. F. Erbacher, and L. B. Feldman. Multidimensional data visualization. Behavior Research Methods, Instruments, \&amp; Computers, 34(2):158-162, 2002.

[31] J. Pearl. Causality. Cambridge University Press, 2009.

[32] R. A. Rensink and G. Baldridge. The perception of correlation in scatterplots. In Computer Graphics Forum, vol. 29, pp. 1203-1210. Wiley Online Library, 2010.

[33] G. Robertson, R. Fernandez, D. Fisher, B. Lee, and J. Stasko. Effectiveness of animation in trend visualization. IEEE Transactions on Visualization and Computer Graphics, 14(6):1325-1332, 2008.

[34] M. E. Roser, J. A. Fugelsang, K. N. Dunbar, P. M. Corballis, and M. S. Gazzaniga. Dissociating processes supporting causal perception and causal inference in the brain. Neuropsychology, 19(5):591, 2005.

[35] H. Rosling and Z. Zhang. Health advocacy with gapminder animated statistics. Journal of epidemiology and global health, 1(1):11-14, 2011.

[36] A. Schlottmann, E. D. Ray, A. Mitchell, and N. Demetriou. Perceived physical and social causality in animated motions: Spontaneous reports and ratings. Acta psychologica, 123(1-2):112-143, 2006.

[37] B. J. Scholl and P. D. Tremoulet. Perceptual causality and animacy. Trends in cognitive sciences, 4(8):299-309, 2000.

[38] D. J. Simons and C. F. Chabris. Gorillas in our midst: Sustained inattentional blindness for dynamic events. perception, 28(9):10591074, 1999.

[39] S. Trickett and G. Trafton. A primer on verbal protocol analysis. In J. C. D. Schmorrow and Nicholson, eds., Handbook of virtual environment training, chap. 18. 2007.

[40] B. Tversky, J. B. Morrison, and M. Betrancourt. Animation: can it facilitate? International Journal of Human-Computer Studies, 57(4):247262, 2002.

[41] C. Ware. Information visualization: perception for design. Elsevier, 2012.

[42] C. Ware, E. Neufeld, and L. Bartram. Visualizing causal relations. In Proceedings of IEEE Information Visualization, vol. 99, 1999.

[43] P. A. White. Visual impressions of force exerted by one object on another when the objects do not come into contact. Visual Cognition, 19(3):340-366, 2011.

[44] P. A. White. Visual impressions of causality: Effects of manipulating the direction of the target object's motion in a collision event. Visual Cognition, 20(2):121-142, 2012.

[45] P. C. Wong and R. D. Bergeron. 30 years of multidimensional multivariate visualization. Scientific Visualization, 2:3-33, 1994.

[46] J. S. Yi, R. Melton, J. Stasko, and J. A. Jacko. Dust \& magnet: multivariate information visualization using a magnet metaphor. Information visualization, 4(4):239, 2005 